\title{
PLAN DE MANEJO AMBIENTAL PARA LA INDUSTRIA LADRILLERA, CASO ASOCIACIÓN DE LADRILLEROS DE PITALITO HUILA "ASOLAPIH"
}

\section{ENVIRONMENTAL MANAGEMENT PLAN FOR BRICKLYER INDUSTRY, CASE ASOCIACIÓN DE LADRILLEROS DE PITALITO HUILA “ASOLAPIH”}

\author{
Deya Maritza Cortes Enríquez ${ }^{1 *}$, Martha Adriana Peña Torres ${ }^{2}$, Tatiana Parra Tejada ${ }^{3}$ \\ ${ }^{1}$ Instructora, SENA. Grupo de investigación Yamboró. maritzacortes@misena.edu.co \\ ${ }^{2}$ Instructora SENA. Grupo de investigación Yamboró. mapenat@ misena.edu.co \\ 3 SENA. Grupo de investigación Yamboró. tparra5@misena.edu.co
}

\section{Resumen}

Mediante el desarrollo de competencias de aprendizaje de evaluación de impacto ambiental aprendices e instructores SENA, evaluaron el escenario ambiental de doce ladrilleras tradicionales en el municipio de Pitalito Huila, resaltando el proceso organizacional que ha desarrollado el gremio ladrillero de la zona de estudio, al constituir la Asociación de Ladrilleros de Pitalito Huila Asolapih. La evaluación se centró en los aspectos ambientales y socioeconómicos, teniendo en cuenta las fases del proceso productivo, mediante visitas de inspección en campo se logró desarrollar diagnóstico ambiental, el cual fue la herramienta principal para valorar los impactos mediante la metodología de Conesa Fernández. Posteriormente, se formularon estrategias de manejo ambiental enfocadas en la prevención, mitigación o compensación de dichos impactos. Es por esto que la Asociación de ladrilleros actualmente cuenta con proyectos que pueden compensar la problemática por deterioro social y ambiental que ella genera, además se encuentran en proceso de licenciamiento ambiental para el área de reserva especial de minería tradicional, lo cual genera un mayor compromiso con el cumplimiento de la normatividad ambiental vigente. Se deberán implementar medidas tales como la construcción de hornos ecológicos, educación ambiental, gestión integral de residuos sólidos y plan de inversión social.

Palabras clave: Impacto ambiental, Ladrillera, Pitalito.

\begin{abstract}
Through the development of environmental impact assessment competencies, SENA apprentices and instructors, evaluated the environmental scenario of twelve traditional brick factories in the municipality of Pitalito Huila, highlighting the organizational process that the bricklayer has developed in the municipality of Pitalito, by constituting the Association of Ladrilleros de Pitalito Huila Asolapih. The evaluation focused on the environmental and socioeconomic aspects, taking into account the phases of the production process. Through inspection visits in the field, the environmental diagnostic development was achieved, which It was the main tool to assess the impacts of the methodology of Conesa Fernández, then environmental management strategies are formulated focused on the prevention, mitigation or compensation of said impacts. That is why the Bricks Association now has projects that can compensate the problems of social and environmental deterioration that it generates, in addition to the fact that there is an environmental licensing process for the special reserve area of Traditional Mining, which generates a greater commitment to compliance with current environmental regulations. It should be
\end{abstract}


implemented such as the construction of ecological furnaces, environmental education, solid waste management and social investment plan.

Keywords: environmental impact, brick factory,Pitalito.

\section{Introducción}

El municipio de Pitalito se encuentra en el sur del Departamento del Huila y es eje comercial surcolombiano debido a su ubicación estratégica, la industria ladrillera es una de las principales fuentes de empleo en esta región, pero de igual forma es una actividad que genera impacto ambiental significativo, ya que se está afectando recursos naturales como el aire y el suelo. Con este trabajo se busca valorar los impactos ambientales generados en el sector ladrillero del municipio de Pitalito para formular e implementar medidas de manejo ambiental para controlar dichos impactos y que el sector pueda trabajar en armonía con el medio ambiente teniendo en cuenta las características de la zona, la cual cuenta con grandes depósitos ricos en arcilla apta para la elaboración de diferentes tipos de ladrillo.

Para formular el plan de manejo ambiental se realizó la caracterización del proceso productivo, un diagnóstico ambiental y se identificaron los agentes que intervienen para conocer el tipo de contaminación generada por esta industria y también los efectos positivos que genera esta industria en la región. Se pretende brindar una herramienta de manejo ambiental, Por ello, es importante mejorar los procesos de explotación y de producción mediante la tecnificación y sus buenas prácticas que contribuyen a mitigar parte de los efectos dañinos (Sánchez, et al, 2013).

\section{Metodología}

Para la obtención de la información se utilizaron fuentes primarias y secundarias, se realizaron visitas técnicas a cada una de las 12 fábricas objeto de estudio donde se identificaron aspectos asociados a la producción del ladrillo y las acciones susceptibles de producir impactos ambientales, para esta etapa se contó con el apoyo del gremio de ladrilleros, además se identificaron características generales de la zona asociadas a los componentes bióticos abióticos y se contó con información secundaria, principalmente obtenida del Atlas ambiental de la biodiversidad del municipio de Pitalito, Plan de Ordenamiento Territorial, Plan de Ordenación y manejo de la cuenca del río Guarapas, posteriormente se valoraron los impactos ambientales mediante la metodología Conessa Fernández y finalmente se formuló el plan de manejo ambiental para establecer las medidas preventivas, de mitigación y de compensación.

\subsection{Contexto Geográfico}

El municipio de Pitalito está constituido por ocho corregimientos y 136 veredas, las ladrilleras objeto de estudio se encuentran en los corregimientos de Criollo, en las veredas de Criollo, Llano Grande, Contador, Solarte y Santa Rosa. Estas fábricas se encuentran dentro del área de reserva especial otorgada por el Ministerio de Minas.

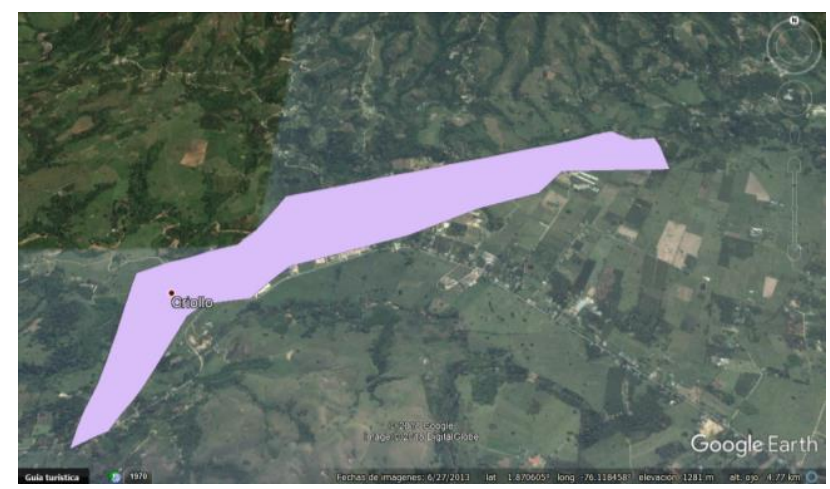

Figura. 1. Ubicación Geográfica Corregimiento de Criollo, Pitalito Huila.

\section{Resultados}

\subsection{Producción de ladrillo}


45

La producción se desarrolla a través de 21 hornos agrupados en 12 fábricas, dichas empresas son: Aránzazu, ARVA, Buenos Aíres, Cazador, Contador, ILAPI, J8, La Chaquira, Nevito, Llano Grande, Manantial y Villa Laura. Este sector genera 150 empleos directos, beneficiando a familias de la región.

La capacidad promedio de los hornos tradicionales es de 10.000 ladrillos, teniendo en cuenta que en su mayoría se produce ladrillo tipo farol, estos hornos tienen una antigüedad de media de 15 años, el estado de estos hornos es regular y de funcionamiento artesanal, por lo cual la autoridad ambiental exige la conversión tecnológica a hornos ecológicos.

A nivel general el proceso desarrolla las etapas de extracción de la materia prima (arcilla) que se obtiene de minas ubicadas en el mismo corregimiento de Criollo, estas minas actualmente se encuentran en proceso de licenciamiento ambiental, luego de ser declarada el área de reserva especial para minería tradicional, dentro de la cual se encuentran 9 minas de arcilla. Posteriormente este material es almacenado en los patios de acopio, luego se procede a la etapa de molienda y mezclado de la arcilla, extrusión, moldeo y corte, secado, cocción, almacenamiento y comercialización. Estas etapas para el estudio de denominaron Acciones Susceptibles de Producir Impacto Ambiental (ASPI)

Los factores del clima son factor importante en la producción ladrillera. Así, en los meses con pocas precipitaciones se realizan aproximadamente 4 quemas por mes, y en los meses con altos niveles de lluvias, 3 quemas aproximadamente, con una duración de 24 horas en cada quema.

En cuanto a los materiales de combustión, se usa carbón mineral o térmico, el cual es comprado por toneladas y transportado desde otras regiones como Boyacá y Cundinamarca, en ocasiones también se usa cascarilla de café que se obtiene de las trilladoras de la región.

Con relación a la calidad del aire, en ninguna de las fábricas se ha realizado estudio de emisiones atmosféricas, por lo cual se desconoce la concentración de emisiones generada por este sector en el municipio de Pitalito.

También es importante resaltar que existe clara conciencia de los productores acerca de su posición ante las autoridades ambientales, por lo cual se encuentran realizando las gestiones pertinentes para la construcción de hornos ecológicos, una de sus estrategias ha sido conformar la Asociación y desde allí han establecido sus prioridades para gestionar sus problemas con las diferentes instancias gubernamentales.

\subsection{Aspectos ambientales e Impactos ambientales}

De acuerdo a las condiciones del proceso desarrollado y las condiciones ambientales de la zona, se logró identificar las Acciones Susceptibles de Producir Impacto Ambiental (ASPI), los componentes impactados y se identificaron aspectos e impactos ambientales que fueron valorados mediante la matriz de impacto ambiental Conessa Fernandez (Arboleda, 2005), en la siguiente tabla se resumen los aspectos e impactos identificados.

Tabla 1. Aspectos e impactos ambientales sector ladrillero

\begin{tabular}{|c|c|c|}
\hline $\begin{array}{c}\text { Aspecto } \\
\text { Ambiental }\end{array}$ & $\begin{array}{c}\text { Impacto } \\
\text { Ambiental }\end{array}$ & $\begin{array}{c}\text { Importancia } \\
\text { Ambiental }\end{array}$ \\
\hline $\begin{array}{l}\text { Demanda de } \\
\text { obra calificada y } \\
\text { no calificada de } \\
\text { propietarios y } \\
\text { empleados. }\end{array}$ & $\begin{array}{l}\text { Mejoramiento } \\
\text { del nivel y } \\
\text { calidad de vida } \\
\text { de la población. }\end{array}$ & $\begin{array}{l}\text { CRITICO } \\
\text { (POSITIVO) }\end{array}$ \\
\hline $\begin{array}{l}\text { Generación de } \\
\text { ruido. }\end{array}$ & $\begin{array}{l}\text { Contaminación } \\
\text { Atmosférica }\end{array}$ & CRITICO \\
\hline
\end{tabular}




\begin{tabular}{|c|c|c|}
\hline $\begin{array}{l}\text { Generación de } \\
\text { material } \\
\text { particulado. } \\
\text { Emisiones } \\
\text { Atmosféricas } \\
\text { Consumo de } \\
\text { combustible. }\end{array}$ & & (NEGATIVO) \\
\hline $\begin{array}{l}\text { Gasto } \\
\text { energético. }\end{array}$ & $\begin{array}{l}\text { Agotamiento de } \\
\text { los recursos } \\
\text { naturales }\end{array}$ & $\begin{array}{l}\text { MODERADO } \\
\text { (NEGATIVO) }\end{array}$ \\
\hline $\begin{array}{l}\text { Consumo de } \\
\text { agua }\end{array}$ & $\begin{array}{l}\text { Agotamiento de } \\
\text { recurso hídrico }\end{array}$ & $\begin{array}{l}\text { IRRELAVANTE } \\
\text { (NEGATIVO) }\end{array}$ \\
\hline $\begin{array}{l}\text { Generación de } \\
\text { residuos sólidos. } \\
\text { Uso del suelo }\end{array}$ & $\begin{array}{l}\text { Cambio en las } \\
\text { propiedades } \\
\text { fisicoquímicas } \\
\text { del suelo. } \\
\text { Afectación a } \\
\text { flora y fauna }\end{array}$ & $\begin{array}{c}\text { CRITICO } \\
\text { (NEGATIVO) }\end{array}$ \\
\hline
\end{tabular}

Fuente: propia

De acuerdo a la valoración, se encontró que los impactos calificados como críticos (Valor de importancia mayor a 75) son la contaminación del aire y la alteración de los suelos, lo cual se debe a la cocción del ladrillo en hornos artesanales que no cuentan con sistemas de control de emisiones y a la explotación de la arcilla en las minas, ya que se están alterando las condiciones fisicoquímicas de los suelos.

\subsection{Medidas de manejo ambiental}

De acuerdo a la significancia de los impactos, se proponen las siguientes medidas de manejo ambiental, teniendo en cuenta los términos de referencia establecidos para los estudios de impacto ambiental para proyectos de minería (Minambiente, 2016):

Tabla 2. Listado de programas de manejo

\begin{tabular}{cl}
\hline $\begin{array}{c}\text { Componente } \\
\text { ambiental }\end{array}$ & \multicolumn{1}{c}{ Programa de manejo } \\
\hline & Manejo de suelo \\
& Manejo de estériles y escombros \\
& Manejo del recurso hídrico
\end{tabular}

Abiótico
Manejo del recurso aire (Construcción de hornos ecológicos)

Manejo integral de residuos solidos

Manejo del paisaje

\begin{tabular}{ll} 
Biótico & $\begin{array}{l}\text { Programa de restauración, rehabilitación y } \\
\text { recuperación de áreas intervenidas. } \\
\\
\text { Conservación de ecosistemas naturales y de } \\
\text { la oferta de servicios ecosistémicos. }\end{array}$ \\
\hline Social & Programa de manejo de atención, \\
& información y participación comunitaria. \\
& Contratación de mano de obra local. \\
& Capacitación y educación a la comunidad.
\end{tabular}

Fuente: propia

\section{Conclusiones}

La expansión de la industria ladrillera, sumada a la organización empresarial y gremial, permite desarrollar un trabajo colaborativo que permita tecnificar el mediante estrategias de producción limpia en el municipio de Pitalito.

Debido a la afectación generada al recurso aire, es necesario que se implementen estrategias de producción limpia, tal como la reconversión tecnológica a hornos ecológicos mitigando de esta forma el impacto ambiental negativo y generando cumplimiento de la normatividad ambiental vigente.

El proceso de fabricación de ladrillos genera impacto ambientales moderados, críticos e irrelevantes, tal como se ha conocido en otros estudios (Valverde et al, 2004), por ende se requiere de la implementación de medidas de manejo preventivas, de mitigación y compensatorias.

\section{Agradecimientos}

Al SENA por brindar el apoyo al grupo de aprendices del Tecnologo en Control Ambiental, Agua y saneamiento y Técnico en Manejo Ambiental. Al líder SENNOVA Juan José 
47

Villlaquirán y a la dinamizadora de Tecnoparque Dayhana Benavides por el apoyo incondicional

A la Asociación de ladrilleros de Pitalito Huila, por disponibilidad y apoyo en el desarrollo del estudio.

\section{Referencias Bibliografícas}

Arboleda, J. (2005). Manual para la evaluación de impacto ambiental de proyectos, obras o actividades. Medellín: Empresas públicas de Medellín.

Ministerio de Ambiente Desarrollo Sostenible. (2016). Términos de referencias para estudios de impacto ambiental para proyectos de minería. Recuperado de http://www.anla.gov.co/sites/default/files /comunicaciones/SIPTA/Terminos_refer encia/tr_eia_mineria_2016.pdf

Valverde, M., Bances, E., Rojas A. \& Rodriguez, B. (2004). Impacto ambiental producido por la fabricacion de Ladrillos en el valle del alto mayo- San Martin. Programa Ingeniería Ambiental. Universidad de Pamplona.

Romo Aguilar, María de Lourdes, Córdova Bojórquez, Gustavo, \& Cervera Gómez, Luis E. (2004). Estudio urbano-ambiental de las ladrilleras en el municipio de Juárez. Estudios fronterizos, 5(9), 9-34. Recuperado de http://www.scielo.org.mx/scielo.php?scri $\mathrm{pt}=\mathrm{sci} \_$arttext\&pid=S0187-

$69612004000100001 \&$ lng=es\&tlng=es.

Silva, M. S., \& Valencia, L. A. Z. (2013). Impacto ambiental y gestión del riesgo de ladrilleras en la vereda Los Gómez de Itagüí. Cuaderno Activa, (5), 109-123.
D.M. Cortes Enríquez, Ingeniero Ambiental de la Universidad del Cauca; Especialista tecnológica en Gestión Ambiental (2014) del SENA. Instructora Centro de Gestión y Desarrollo Sostenible Surcolombiano, Integrante activo del Grupo de Investigaciones Yamboró.

M.A. Peña Torres, Química ,de la Universidad del Quindío, Maestría en Sistemas Sostenibles de producción de la universidad de la Amazonia. Especialista en producción de bioinsumos y extractos vegetales CGDSS. Instructora Centro de Gestión y Desarrollo Sostenible Surcolombiano. Docente Universidad CORHUILA Corporación Universitaria del Huila. Tutora UNAD Universidad Nacional Abierta y a distancia. Investigador activo del Grupo de investigación YAMBORÓ.

\footnotetext{
*Para citar este artículo: Cortes Enríquez DM .;Peña Torres MA.;, Parra Tejada T.

Environmental management plan for bricklyer industry,case asociación de ladrilleros de Pitalito Huila. "ASOLAPIH".

Revista Bistua.2017.15(2):43-47.
}

+ Autor para el envió de correspondencia y la solicitud de las separatas: Deya Maritza Cortes Enríquez . Instructora, SENA. Grupo de investigación Yamboró.
Recibido: Octubre 22 de 2016

Aceptado: Enero 30 de 2017 